\title{
Selection of the artificial pollution procedure for polymer isolation in the conditions of the atmospheric environment in Cuba
}

\author{
Ramón G. Guevara ${ }^{1}$, José A. M. Barbado ${ }^{2}$, Raiza J. Quiñones ${ }^{3}$ and Miguel A. R. Moreno ${ }^{4}$ \\ ${ }^{1,2,3,4}$ Centro de Investigaciones y Pruebas Electroenergéticas (CIPEL). Instituto Superior Politécnico José Antonio Echeverría (CUJAE). \\ Havana, Cuba.
}

Email:ramon@electrica.cujae.edu.cu,jamb@electrica.cujae.edu.cu,rjarel@electrica.cujae.edu.cu,mangel@electrica.cujae.edu.cu

Received: November $08^{\text {th }}, 2017$.

Accepted: January $11^{\text {th }}, 2018$.

Published: March $13^{\text {th }}, 2018$.

Copyright (C2016 by authors and Institute of Technology Galileo of Amazon (ITEGAM).

This work is licensed under the Creative Commons Attribution International

License (CC BY 4.0).

http://creativecommons.org/licenses/by/4.0/ (c) (i) (9) Opean Actes:

\begin{abstract}
In the work different variants of artificial contamination in the polymeric isolation are analyzed using the technique of the deposited layer, keeping constant the amount of inert substance and varying the quantities of active substance in each solution and the amounts of pollutants applied on the surface of the insulators For each of these variants, the Equivalent Density of Deposited Salt (DESD) is determined to the polluting solutions obtained. The results obtained are processed, using statistical techniques, in order to compare them with the classification already established in the specific conditions imposed by the atmospheric environment in our country and select the variant that most closely matches the requirements of natural pollution levels. Finally, the procedure is elaborated detailing the requirements of the selected contamination technique and highlights those aspects considered most important to achieve satisfactory results.
\end{abstract}

Keywords: Artificial Contamination; Equivalent Density of Deposited Salt (DESD); Statistical Techniques.

\section{Selección del procedimiento de contaminación artificial para el aislamiento polimérico en las condiciones del medio atmosférico en Cuba}

\section{RESUMEN}

En el trabajo se analizan diferentes variantes de contaminación artificial en el aislamiento polimérico utilizando la técnica de la capa depositada, manteniendo constante la cantidad de sustancia inerte y variando las cantidades de sustancia activa en cada solución y las cantidades de contaminantes aplicadas en la superficie de los aisladores. Para cada una de estas variantes se determina la densidad equivalente de sal depositada (desd) a las soluciones contaminantes obtenidas. Se procesan los resultados obtenidos, utilizando técnicas estadísticas, con el objetivo de compararlos con la clasificación ya establecida en las condiciones especificas que impone el medio atmosférico en nuestro país y se selecciona la variante que más se acerque a las exigencias de los niveles de contaminación natural. Por ultimo, se elabora el procedimiento donde se detallan los requerimientos de la técnica operatoria de contaminación seleccionada y se destacan aquellos aspectos considerados de mayor importancia para lograr resultados satisfactorios.

Palabras Claves: Contaminación Artificial; Densidad Equivalente de Sal Depositada (DESD); Técnicas Estadísticas

\section{INTRODUCCIÓN}

La validez de la hipótesis teórica que plantea que independientemente del origen y la combinación de contaminantes atmosféricos en cualquier región, los mismos pueden ser representados artificialmente por la mezcla de una sustancia inerte responsable de sus cualidades adhesivas y absorbentes y otra eléctricamente activa que al humedecerse se 
disocia en iones, proporcionándole a la capa de contaminación sus cualidades conductivas, significo un gran avance en los estudios de contaminación en condiciones de laboratorio.

Fue posible, a partir de este momento, preparar soluciones con contaminación artificial, realizar ensayos y obtener mayor número de resultados en menor tiempo, quedando solo por resolver la cuestión de lograr la mejor simulación de las condiciones de contaminación natural, donde la reproducibilidad y representatividad de los resultados tienen un papel protagónico. La introducción del aislamiento polimérico ha demostrado que los polímeros utilizados, hasta estos momentos, presentan problemas en condiciones específicas de operación.

Algunos de los fenómenos perjudiciales en este tipo de aislamiento tienen su origen en las condiciones superficiales del aislador, a saber altos niveles de incidencia de rayos ultravioleta, alto contenido de humedad, descargas por contaminación, etc. Otros fenómenos, debido al mayor número de interfases tienen su explicación interna [1-4]. El desarrollo de estos fenómenos perjudiciales disminuyen las propiedades aislantes y los requerimientos mecánicos llegando a situaciones extremas en las cuales el aislador falla.

Los ensayos artificiales en el aislamiento polimérico permiten simular diferentes condiciones, tales como:

diferentes regimenes de humedecimientos, calentamientos prolongados de su superficie, lluvias de diferentes intensidades, incidencia de nieblas salinas, radiación solar, estudio de variables sinérgicas, diferenciar comportamientos de aisladores en ambientes de igual severidad, entre otros. Algunas normas relacionadas con esta temática son referenciadas en [5-7].

La IEC y otras instituciones trabajan en la dirección de lograr resultados que permitan una simulación mas representativa de los fenómenos que perjudican el comportamiento de los aisladores en condiciones de operación y la comunidad científica internacional, relacionada con esta temática junto con los fabricantes persiguen la estrategia central de obtener nuevos compuestos que sean capaces de soportar disímiles y severas condiciones de operación.

Nuestro país, se caracteriza por tener un clima tropical húmedo y condiciones especificas metereológicas que pueden resultar extremas en distintas épocas del año, tales como: alta incidencia de rayos ultravioleta, regímenes severos y prolongados de humedecimiento, influencia directa de aerosoles marinos, fuertes vientos huracanados, alta incidencia de descargas atmosféricas, etc.

Es por ello que en el trabajo se realizan esfuerzos en el sentido especifico de lograr la mejor representación de nuestros niveles de contaminación del aislamiento en el laboratorio utilizando la técnica de la capa predepositada [8] y evaluando, por medio de la DESD [9], el nivel de contaminación para de esta forma poder determinar com más exactitud la influencia de la intensidad de la contaminación atmosférica.

\section{METODOLOGÍA DE INVESTIGACIÓN}

En todas las soluciones contaminantes preparadas para la ejecución de las variantes de ensayos se mantienen constantes las cantidades de material inerte, utilizando $40 \mathrm{~g}$ de caolín y un litro de agua desmineralizada, variando las cantidades de cloruro de sodio, utilizado como material activo.
En cada una de las soluciones preparadas para simular las condiciones de contaminación natural se practican 3 variantes de ensayos diferenciándose entre si por las cantidades de material activo añadido según se muestra em la tabla 1.

Tabla 1: Variantes de ensayos para las soluciones contaminantes

\begin{tabular}{|c|c|c|c|}
\hline $\begin{array}{c}\text { Soluciones } \\
\text { Contaminantes }\end{array}$ & \multicolumn{3}{|c|}{ Substancia activa } \\
\hline 1 & 0,8 & 1,0 & 1,2 \\
\hline 2 & 2,0 & 3,2 & 4,8 \\
\hline 3 & 6,2 & 8,0 & 9,4 \\
\hline 4 & 11,2 & 14,0 & 16,2 \\
\hline
\end{tabular}

Fonte: Autores, (2018).

Los ensayos se realizan sobre el aislador que se muestra a continuación en la figura 1.

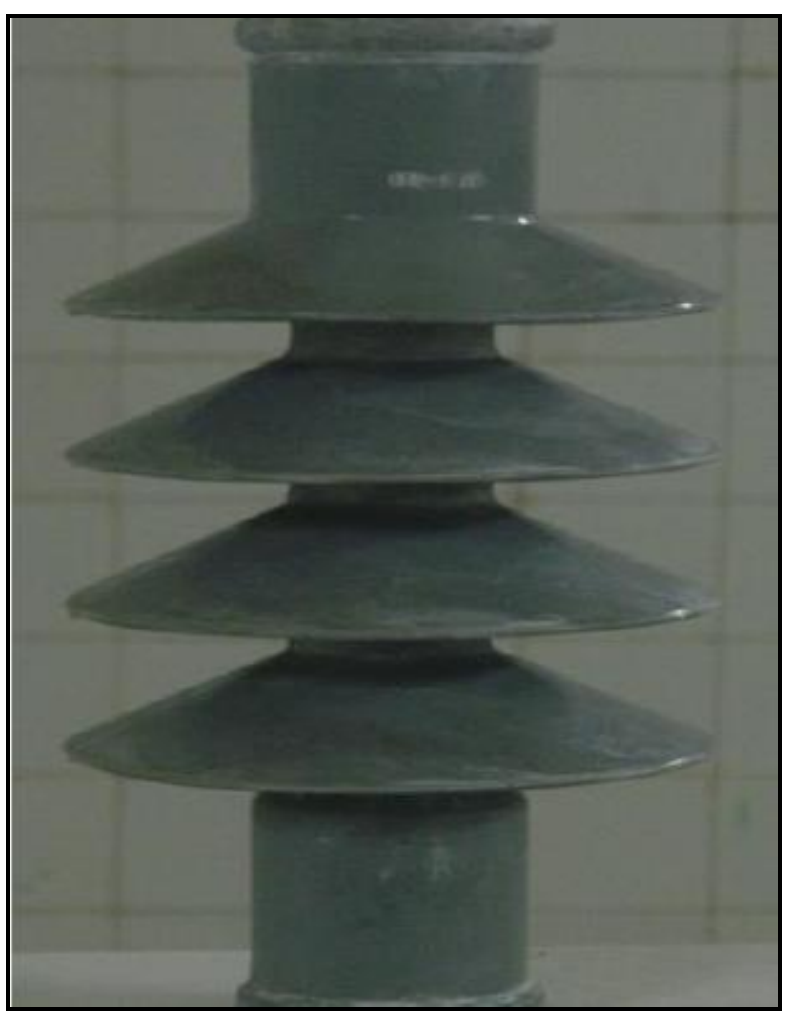

Figura 1: Aislador ensayado.

Fonte: Autores, (2018).

Se definen las variables involucradas en el experimento como:

- Variable dependiente: DESD.

- Variables independientes: Temperatura, conductividad electrolítica de la solución contaminante, medios de medición, el aislador seleccionado, personal que realiza el ensayo.

Planteamiento de la hipótesis Ho.

No existen diferencias significativas entre los valores de DESD, cuando se varía en pequeñas cantidades la sustancia activa de las soluciones de contaminación artificial para las zonas estudiadas.

Las mediciones de conductividad electrolítica se realizan con un conductímetro digital GMH 3410. Con el valor de conductividad referido a $20^{\circ} \mathrm{C}$, el área de la superficie lavada del 
aislador y el volumen de agua empleado, se calcula el valor de DESD por medio de las expresiones (1) y (2).

$$
S_{a}=\left(5.7 * \Gamma_{20^{\circ} C}\right)^{1.03}
$$

Donde:

$\mathrm{S}_{\mathrm{a}}$ : Salinidad de la solución $(\mathrm{kg} / \mathrm{m} 3)$.

$\Gamma_{20^{\circ} \mathrm{C}}$ : Conductividad electrolítica referida a $20^{\circ} \mathrm{C}(\mathrm{S} / \mathrm{m})$.

$$
D E S D=\frac{S_{a} \times V}{A}
$$

Donde:

A- Área lavada del aislador $\left(\mathrm{cm}^{2}\right)$.

$\mathrm{V}$ - Volumen de la solución $\left(400 \mathrm{~cm}^{3}\right)$.

\section{PROCESAMIENTO ESTADÍSTICO - INTERPRETACIÓN DE LOS RESULTADOS}

El análisis de varianza (ANOVA) [10] se expresa mediante la aplicación del estadístico de Fisher, el cual permite indagar sobre la existencia o no de diferencias estadísticamente significativas entre los valores medios de DESD para las cantidades de sustancia activa empleadas. En el análisis estadístico se realiza la exploración de los datos utilizándose las medidas resumen y se verifican las premisas del ANOVA, como la aleatoriedad e independencia de las observaciones, homogeneidad de la varianza y normalidad de la muestra de datos experimentales obtenidos. En todos los casos se cumplieron las premisas.

Las tablas de la 2 a la 7 muestran los resultados del ANOVA para las cuatro soluciones estudiadas.

Tabla 2: Análisis de varianza de la solución No. 1.

\begin{tabular}{|c|c|c|c|c|c|}
\cline { 2 - 6 } \multicolumn{1}{c|}{} & $\begin{array}{c}\text { Suma de } \\
\text { cuadrados }\end{array}$ & g.l & $\begin{array}{c}\text { Cuadrado } \\
\text { medio }\end{array}$ & F & Sig. \\
\hline $\begin{array}{c}\text { Entre } \\
\text { Grupos }\end{array}$ & $7,5 \times 10^{-5}$ & 2 & $3,8 \times 10^{-5}$ & 2,01 & 0,168 \\
\hline $\begin{array}{c}\text { Dentro } \\
\text { de } \\
\text { grupos }\end{array}$ & $2,8 \times 10^{-4}$ & 15 & $1,9 \times 10^{-5}$ & & \\
\hline Total & $3,6 \times 10^{-4}$ & 17 & & & \\
\hline
\end{tabular}

*gl: Grados de libertad.

Fonte: Autores, (2018).

Tabla 3: Análisis de varianza de la solución No. 2.

\begin{tabular}{|c|c|c|c|c|c|}
\cline { 2 - 6 } \multicolumn{1}{c|}{} & $\begin{array}{c}\text { Suma de } \\
\text { cuadrados }\end{array}$ & g.l & $\begin{array}{c}\text { Cuadrado } \\
\text { medio }\end{array}$ & F & Sig. \\
\hline $\begin{array}{c}\text { Entre } \\
\text { Grupos }\end{array}$ & $4,86 \times 10^{-4}$ & 2 & $2,43 \times 10^{-4}$ & 7,93 & 0,05 \\
\hline $\begin{array}{c}\text { Dentro } \\
\text { de } \\
\text { grupos }\end{array}$ & $4,29 \times 10^{-4}$ & 14 & $3,06 \times 10^{-5}$ & & \\
\hline Total & $9,15 \times 10^{-4}$ & 16 & & & \\
\hline
\end{tabular}

Fonte: Autores, (2018).
Tabla 4: Prueba de Duncan.

\begin{tabular}{|c|c|c|c|}
\hline \multicolumn{2}{|c|}{ Prueba de Duncan } & \multicolumn{2}{c|}{$\boldsymbol{\alpha}=\mathbf{0 , 0 5}$} \\
\hline Cod 2 & $\mathrm{N}$ & 1 & 2 \\
\hline $\mathbf{1}$ & 5 & $2,06 \times 10^{-2}$ & \\
\hline $\mathbf{2}$ & 6 & $2,17 \times 10^{-2}$ & \\
\hline $\mathbf{3}$ & 6 & & $3,23 \times 10^{-2}$ \\
\hline Significación & & 0,751 & 1,00 \\
\hline
\end{tabular}

Fonte: Autores, (2018).

Tabla 5: Análisis de varianza de la solución No. 3.

\begin{tabular}{|c|c|c|c|c|c|}
\cline { 2 - 6 } \multicolumn{1}{c|}{} & $\begin{array}{c}\text { Suma de } \\
\text { cuadrados }\end{array}$ & g.l & $\begin{array}{c}\text { Cuadrado } \\
\text { medio }\end{array}$ & F & Sig. \\
\hline $\begin{array}{c}\text { Entre } \\
\text { Grupos }\end{array}$ & $2,74 \times 10^{-4}$ & 2 & $1,4 \times 10^{-4}$ & 3,763 & 0,047 \\
\hline $\begin{array}{c}\text { Dentro } \\
\text { de } \\
\text { grupos }\end{array}$ & $5,47 \times 10^{-4}$ & 15 & $3,6 \times 10^{-5}$ & & \\
\hline Total & $8,21 \times 10^{-4}$ & 17 & & & \\
\hline
\end{tabular}

Fonte: Autores, (2018).

Tabla 4: Prueba de Duncan.

\begin{tabular}{|c|c|c|c|}
\hline \multicolumn{2}{|c|}{ Prueba de Duncan } & \multicolumn{2}{c|}{$\alpha=0,05$} \\
\hline Cod 3 & N & 1 & 2 \\
\hline 1 & 6 & $4,16 \times 10^{-2}$ & \\
\hline 2 & 6 & $4,35 \times 10^{-2}$ & $4,35 \times 10^{-2}$ \\
\hline 3 & 6 & & $5,07 \times 10^{-2}$ \\
\hline Significación & & 0,594 & 0,058 \\
\hline
\end{tabular}

Fonte: Autores, (2018).

Tabla 7: Análisis de varianza de la solución No. 4.

\begin{tabular}{|c|c|c|c|c|c|}
\cline { 2 - 6 } \multicolumn{1}{c|}{} & $\begin{array}{c}\text { Suma de } \\
\text { cuadrados }\end{array}$ & g.l & $\begin{array}{c}\text { Cuadrado } \\
\text { medio }\end{array}$ & F & Sig. \\
\hline $\begin{array}{c}\text { Entre } \\
\text { Grupos }\end{array}$ & $1,5 \times 10^{-4}$ & 2 & $7,6 \times 10^{-5}$ & 1,86 & 0,19 \\
\hline $\begin{array}{c}\text { Dentro } \\
\text { de } \\
\text { grupos }\end{array}$ & $6,2 \times 10^{-4}$ & 15 & $4,1 \times 10^{-5}$ & & \\
\hline Total & $7,7 \times 10^{-4}$ & 17 & & & \\
\hline
\end{tabular}

Fonte: Autores, (2018).

En los resultados mostrados se observa que no existen diferencias estadísticamente significativas en las soluciones 1 y 4 con un $95 \%$ de confiabilidad, al ser la Sig. mayor que el nivel de significación fijado para el error $(\alpha=0,05)$. Lo que representa que entre los valores de DESD promedio obtenidos a partir de variar la cantidad de la componente activa en las tres variantes de ensayos no difieren apreciablemente entre sí, aceptándose en estos casos la hipótesis Ho.

En el caso de las soluciones 2 y 3 si se detectan diferencias estadísticamente significativas entre los valores de DESD promedio, por lo que fue necessário recurrir a la prueba de Duncan con el objetivo de diferenciar entre que DESD promedio estas se manifestaban. 
Para la solución 2 no se observan diferencias entre las variantes de ensayo de 2,0 y 3,2 g de material activo, pero ambas si muestran diferencias respecto a la variante 3 (tabla 4 ).

Por otra parte, la solución 3 muestra un comportamento que difiere de la solución 2. Las variantes de ensayo de 6,2 y 9,4 $\mathrm{g}$ de material activo difieren entre sí significativamente, pero a su vez ambas no se diferencian de la variante $8,0 \mathrm{~g}$ (tabla 6).

En base al análisis anterior y considerando que desde el punto de vista práctico es más aconsejable realizar el pesaje de mayores cantidades de sustancias buscando una mayor exactitud, se decide seleccionar como cantidades de material activo para la preparación de las diferentes soluciones las indicadas en la tabla 8. En tal sentido, este resultado se encuentra en concordancia com los intervalos de DESD determinados en las condiciones de contaminación natural en nuestro país [11].

Tabla 8: Análisis de varianza de la solución No. 2.

\begin{tabular}{|c|c|c|}
\hline $\begin{array}{c}\text { Soluciones } \\
\text { contaminantes }\end{array}$ & $\begin{array}{c}\text { Sustancia activa } \\
\text { NaCl }(\mathbf{g})\end{array}$ & $\begin{array}{c}\text { Niveles de } \\
\text { Contaminación } \\
\text { DESD }\left(\mathbf{m g} / \mathbf{c m}^{2}\right)\end{array}$ \\
\hline 1 & 1,2 & $\leq 0,02$ \\
\hline 2 & 3,2 & $0,02-0,04$ \\
\hline 3 & 8 & $0,04-0,06$ \\
\hline 4 & 16,2 & $0,06-0,08$ \\
\hline 5 & $>16,2$ & $>0,08$ \\
\hline
\end{tabular}

Fonte: Autores, (2018).

\section{PROPUESTA DE PROCEDIMIENTO DE CONTAMINACIÓN ARTIFICIAL. TÉCNICA OPERATORIA}

La técnica operatoria del procedimiento de contaminación propuesto, consta de siete pasos:

1 - Se lava el aislador cuidadosamente con detergente u outra sustancia apropiada y se enjuaga posteriormente con agua corriente para eliminar cualquier traza de grasa $\mathrm{y} / \mathrm{o}$ polvos.

2 - Se introduce el aislador en un horno eléctrico a uma temperatura de $50-60^{\circ} \mathrm{C}$.

3 - Posteriormente se pone el aislador en la posición de ensayo y se le deposita una capa de contaminante en su superficie, utilizando un pulverizador manual a uma distancia entre 20 - 40 $\mathrm{cm}$ desde la salida del pulverizador y el borde de la aleta del aislador y agitando la solución constantemente para lograr la mayor disolución de sus componentes.

4 - Se deja reposar y enfriar la capa contaminante por um tiempo de 10 a 15 minutos.

5 - Se repiten las instrucciones establecidas en el paso 2.

6 - Se coloca el aislador en la posición de ensayo y se procede a depositar la segunda capa de contaminante tal y como se explica en el paso 3.
7 - Finalmente el aislador debe estar en reposo durante 24 horas antes de proceder al lavado de su superficie y realizar las mediciones y cálculos correspondientes.

\section{CONCLUSIONES}

1. Las mediciones, procesamiento estadístico e interpretación de los resultados realizados muestran como variante más apropiada para simular los niveles de contaminación natural existentes em nuestro país, la que consiste en añadir $1,2 \mathrm{~g}$, $3,2 \mathrm{~g}, 8,0 \mathrm{~g}$ y 16,2 g de sustância activa para la preparación de las soluciones $1,2,3$, y 4 respectivamente. Todas con la adición de 40 g. de caolín como sustancia inerte y la deposición de dos capas de contaminante sobre la superficie de los aisladores.

2. Se elabora un procedimiento de contaminación artificial donde se detalla la técnica operatoria a realizar. Como aspectos importantes se destacan la aplicación de una capa de contaminación base para facilitar la deposición posterior y la necesidad de contar con personal especializado que extreme los cuidados para lograr uma correcta aplicación del contaminante.

3. La simulación y representatividad de las condiciones naturales de contaminación en los ensayos de laboratório significa realizar una correcta ponderación del comportamiento de diferentes tipos de aisladores em distintos niveles de contaminación, lo que se traduce em un impacto económico beneficioso para el sector eléctrico y para el país.

\section{RECOMENDACIÓN}

Utilizando como soporte estadístico las mediciones ejecutadas, se recomienda continuar ampliando el universo de mediciones en otros tipos de aisladores de diferentes configuraciones, para perfeccionar, aun más, la reproducción de las condiciones reales de operación en el laboratorio.

\section{REFERENCIAS}

[1] Fernando M. and Gubanski S. Performance on Nonceramic Insulator under Tropical Field Conditions, IEEE on Power Delivery, Vol. No. 1, 2000.

[2] Kim S-H and Hackman R. Effects on saline-water flow rate and air speed on leakage current in RTV coatings. IEEE Transaction on Power Delivery, Vol. 10 No. 4 October 1995, p.p. 1956-1963.

[3] Gorur R.S. Chang, J,W. Amburguey O.G. Surface hidrophobicity of polymers used for outdoor insulation. IEEE Transantions on Power delivery, Vol. 5, No 4, 1990, p.p. 1923 1928.

[4] Koshino Y. and et. Al. Recovery Behaviour of Hidrophobicity of silicone Rubber for Polymer Insulator Housing Deposited with Contaminant. Transaction on IEE Japan. Vol. 121-A, No.9, 2001.

[5] IEC 61109: 1992. Composite insulators for a.c. overhead lines with a nominal voltage greater than 1000v. Definitions, test methods and acceptation criteria. 
[6] ANSI/IEEE 1024. Recommended practice for specitying distribution composite insulators (suspension type).

[7] ANSI C29.11. Composite suspension insulators for overhead transmission lines with voltage greater than $1000 \mathrm{~V}$.

[8] IEC 60507 “Artificial pollution test on high voltage insulator to be used ac systems", 1991.

[9] CIGRE Insulator Pollution Monitoring. CIGRE Task Force 33.04.03 Electra No.152, February 1994, p.p. 79-90.

[10] Sigarroa, A. Biometría y Diseño Experimental. Parte 1 y 2. Edición Cubana 1985.

[11] Castro F. M., González G. R, Cuervo L. H., Durán V. J.; Mapa de niveles de contaminación del aislamiento de la República de Cuba: trabajo experimental (I), Revista Ingeniería Energética, Vol.XIII, No.3, 1992. ISSN:0534-2608. 